DOI:10.2478/rrlm-2019-0020

\title{
Systemic interleukins levels in community-acquired pneumonia and their association with adverse outcomes
}

\author{
Raluca Elena Tripon ${ }^{1 *}$, Ioana Berindan Neagoe ${ }^{2}$, Livia Budisan², Tudor Lucian \\ Pop $^{3}$, Victor Cristea ${ }^{4}$, Liana Maria Stanca ${ }^{5}$, Mihaela Sorina Lupse ${ }^{1}$
}

1. Department of Infectious Diseases “Iuliu Hatieganu” University of Medicine and Pharmacy, ClujNapoca, Romania; Teaching Hospital of Infectious Diseases Cluj-Napoca, Romania

2. Research Center for Functional Genomics, Biomedicine and Translational Medicine"Iuliu

Hatieganu" University of Medicine and Pharmacy, Cluj-Napoca, Romania

3. Department of Pediatrics, "Iuliu Hatieganu" University of Medicine and Pharmacy, Cluj-Napoca, Romania

4. Department of Immunology, "Iuliu Hatieganu" University of Medicine and Pharmacy, Cluj-Napoca, Romania

5. Faculty of Economics and Business Administration, Business Information Systems Department "Babeş-Bolyai" University of Cluj-Napoca, Romania

\begin{abstract}
Introduction: Community-acquired pneumonia (CAP) is still one of the major causes of morbidity and mortality worldwide. Pro-inflammatory and anti-inflammatory interleukins have been studied to elucidate the role that inflammation plays in its pathogenesis. The aim of this study is to investigate inflammation in CAP, by analyzing in dynamic, serum levels of six interleukins (IL) and their predictive value regarding adverse outcomes. Materials and methods: Forty adult patients with CAP, admitted in the Teaching Hospital of Infectious Diseases, Cluj-Napoca, Romania from December 2015 to February 2017, were enrolled in this study. Serum levels of pro-inflammatory: IL$1 \beta$, TNF- $\alpha, I L-6$, anti-inflammatory: IL-10 and IL-4, along with IL-17A were analyzed in dynamic, on day 1 and day 4.The receiver-operator curves (ROC) were used to analyze the outcome prediction of IL. Results: Serum levels of $I L-1 \beta, I L-6, T N F-\alpha$ and IL-10 have decreased significantly in dynamic, while IL-4 increases. IL-17A has acted like a pro-inflammatory cytokine. We have found a correlation between IL-6 and IL-10 ( $r=0.429, p=0.000), I L-6$ and $I L-17 A(r=0.295, p=0.008)$ and IL-10 and IL-17A $(r=0.475, p=0.000)$. Out of 40 patients, 9 had adverse outcomes, consisting in 9 relapses from which 1 died. IL-6 discriminates alone between adverse and favorable outcomes. With multivariate analysis and multiple regression of all combined $I L$, we have found that there is a predictive model regarding adverse outcomes. Conclusion: IL-10 and IL-17A behave like pro-inflammatory cytokines. IL-6 is a predictive marker for adverse outcomes alone. All IL studied together have an impact on adverse outcomes.
\end{abstract}

*Corresponding author: Raluca Elena Tripon, "Iuliu Hatieganu” University of Medicine and Pharmacy, ClujNapoca, Romania. E-mail: rhorge@yahoo.com; Horge.Raluca@umfcluj.ro 
Keywords: community-acquired pneumonia, interleukins, adverse outcomes

Received: $4^{\text {th }}$ December 2018; Accepted: $3^{\text {rd }}$ March 2019; Published: $15^{\text {th }}$ April 2019

\section{Introduction}

Community-acquired pneumonia (CAP) remains one of the major causes of morbidity and mortality worldwide, despite the advances in diagnostic methods and treatment and it is the leading cause of severe sepsis $[1,2]$. Recent studies have been focused on the role that inflammation plays in the pathogenesis of this disease. Inflammation is part of the host's immune response against infection and if uncontrolled, it can have deleterious consequences for the host $[3,4]$. Several cytokines have been identified in immunopathogenesis of CAP, but most studies that analyzed them, have reported conflicting results and were limited to a single serum determination. IL-1 $\beta$, IL-6, IL-8 and TNF- $\alpha$ as pro-inflammatory, and IL-10 as anti-inflammatory cytokines, have been the most studied in CAP $[1,5]$. IL-4 is another anti-inflammatory cytokine that has been postulated to have important role in allergic diseases, but also in the immune response against $\mathrm{Myco-}$ plasma pneumoniae [6,7]. IL-17A has recently been involved in the immune response during CAP, playing an important role in acute inflammation induced by infectious agents in the airway. $[7,8]$.

This study aimed to investigate inflammation in CAP, by testing serum levels of IL- $1 \beta$, TNF- $\alpha$ and IL- 6 as pro-inflammatory cytokines, IL-10 and IL-4 as anti-inflammatory cytokines and the relationship between them and IL-17A. We studied the interleukin dynamics on admission and on day 4 of hospitalization and their predictive value regarding adverse outcomes.

\section{Materials and methods}

\section{Patients and study design}

A prospective study was carried out from December 2015 to February 2017 in the Teaching
Hospital of Infectious Diseases Cluj-Napoca, Romania and it included adult patients with community-acquired pneumonia defined according to the IDSA [9]. The study protocol was approved by the medical ethics committee of both the Hospital and "Iuliu Hatieganu" University of Medicine and Pharmacy Cluj-Napoca, Romania. A written informed consent was obtained from the patients or closest relatives in each case. Exclusion criteria were represented by: age under 18 years old, pregnancy, HIV infection, active tuberculosis, known malignancies and autoimmune diseases requiring immunosuppressive drugs. Patients were followed-up 90 days after discharge.

Patients were enrolled in the study group within 24 hours of hospital admission. We recorded demographical data, clinical data, length of stay (LOS) in the hospital, various scores for pneumonia severity $[10,11]$, usage of antibiotics prior to hospital admission, presence of complications such as sepsis, according to ACCP/SCCM criteria [12], acute respiratory failure and pleurisy, adverse outcomes during follow-up (relapses or death).

Venous blood samples for cytokine measurements were collected from each patient within the first 24 hours following admission, between 7 and 9 AM, and then again on day 4 of hospitalization. After centrifugation, the serum from patients was split into 2-4 cryotubes and stored at $-70^{\circ} \mathrm{C}$ until it was analyzed.

In order to study the relationship between CAP and inflammation, we analyzed serum levels of cytokines using commercially available enzyme immunoassay technique (ELISA) (IL-1 $\beta$, IL-4, IL-6, IL-10 and IL-17A using BioVendor Research and Diagnostic products, USA; TNF- $\alpha$ using Hycult ${ }^{\circledR}$ biotech assay,USA). The detection limits were $0.3 \mathrm{pg} / \mathrm{ml}$ for IL- $1 \beta, 1.3 \mathrm{pg} / \mathrm{ml}$ 
for IL-4, $0.92 \mathrm{pg} / \mathrm{ml}$ for IL-6, $1.0 \mathrm{pg} / \mathrm{ml}$ for IL-10 and $0.5 \mathrm{pg} / \mathrm{ml}$ for IL-17A.

\section{Statistical analysis}

For statistical analysis, to test for the normality of data, we used Shapiro-Wilk test, for nonparametric data, , the chi square, Wilcoxon, Friedmantests. The Spearman rank correlation test was used in order to assess the relationship between interleukin age and pneumonia severity index. The receiver - operator curves (ROC) with the level of significance set at $\mathrm{p}<0.05$, was used to analyze the accuracy of prediction by estimating the area under the curve. We performed ROC curves using Excell MedCalc 9.6 version and the multivariate analysis with StatMed. For logistic regression (Wald chi, ENTER method) we used SPSS 16.0 (SPSS Inc, Chicago, IL, USA) and MedCalc v12.

\section{Results}

Forty CAP patients were included in this study, with the mean age $63.45 \pm 18.410$ (range 23-87); $27(67.5 \%)$ were men and $23(57.5 \%)$ came from the urban areas. The average LOS in the hospital was $8.58 \pm 3.137$ days, with no significance between gender $(p=0.628)$ or age $(p=0.246)$. Prior to hospitalization, 8 patients had antibiotic treatment within the last 2 weeks. Etiology was established for 10 patients (25\%): 2- Streptococcus pneumoniae, 3- Haemophilus influenza, 1- Haemophilus parainfluenzae, 1- Chlamydia pneumoniae, 1- Serratia marcenscens (from community), 2- Influenza virus (1 A, 1 B).

Regarding complications, 13 patients had respiratory sepsis, 13 pleurisy and 39 had different degrees of acute respiratory failure. The Pneumonia Severity Score (PSI) and CURB-65 were calculated for each patient at admission. Regarding PSI, our patients were included as follows: 2 patients in risk class I, 4 in class II, 5 in class III, 20 in class IV, and 9 in class V.
For CURB-65: 6 patients were included in risk group 1, 21 in group 2 and 13 in group 3. We also used SMART-COP score for 38 of our patients ( 2 cases had incomplete data). The majority ( 24 patients) were included in low risk of needing intensive respiratory or vasopressor support (IRVS), 6 had moderate risk of IRVS, and 8 were considered to have severe CAP (7 with high risk of IRVS and 1 with very high risk).

The serum levels of six cytokines were determined at admission and on day 4 . Their median values and differences between day 1 and day 4 are presented in table 1 [table 1 inserted here]. As shown above, the values of IL-1 $\beta$, IL-6, IL10 and TNF- $\alpha$ decreased significantly between day 1 and day 4. IL-4 values increased significantly on day 4 compared to day 1 , while IL-17A seemed to act as a pro-inflammatory cytokine, but its values remained almost unchanged (figure 1)

There is a positive correlation between IL- $1 \beta$ and IL-6, TNF- $\alpha$, IL-4; IL-6 and TNF- $\alpha$, IL-17A, IL10, IL-4; IL-4 and IL-17A, IL-10; and between IL-10 and IL-17A. (table2)

There is no correlation between patients' age and interleukin levels (IL-1 $\beta$ : $p=0.983$; IL-6: $\mathrm{p}=0.481$; TNF- $\alpha$ : $\mathrm{p}=0.459$; IL-17A: $p=0.268$; IL$4: p=0.656$; IL-10: $p=0.548$ ), nor between gender and interleukin levels (IL-1 $\beta$ : $p=0.062$; IL6: $p=0.461$; TNF- $\alpha: p=0.965$; IL-17A: $p=0.977$; IL-4: $p=0.355$; IL-10: $p=0.170)$.Likewise, we found no significant correlations between PSI risk score and cytokine levels (IL-1 $\beta$ : $p=0.483$; IL-6: $p=0.730 ;$ TNF- $\alpha$ : $p=0.775$; IL-17A: $\mathrm{p}=0.744$; IL-4: $\mathrm{p}=0.112$; IL-10: $\mathrm{p}=0.152$ )Out of the 40 patients under study, 9 (22\%) had adverse outcomes (9 relapses, from which 1 died). We questioned if any interleukin value on day 1 (prior to antibiotic treatment) was predictive for adverse outcomes and the results were that only IL-6 had significant contribution ( $p=0.043$, cut-off $=28.46 \mathrm{pg} / \mathrm{mL}$ ), discriminating between adverse outcomes and favourable outcomes. 
Table 1. Differences between the values of the cytokines on day 1 and day 4

\begin{tabular}{|c|c|c|c|c|c|}
\hline \multirow{2}{*}{ Interleukins } & & \multirow{2}{*}{ Median } & \multirow{2}{*}{ IQR } & \multicolumn{2}{|c|}{ Statistical results } \\
\hline & & & & p-value & Statistic test \\
\hline \multirow{2}{*}{ IL1 $\beta$} & day 1 & 6.025 & 1.506 & \multirow{2}{*}{0.000} & \multirow{2}{*}{$Z=-4.664$} \\
\hline & day 4 & 5.092 & 0.796 & & \\
\hline \multirow{2}{*}{ IL4 } & day 1 & 21.515 & 6.427 & \multirow{2}{*}{0.021} & \multirow{2}{*}{$Z=-2.301$} \\
\hline & day 4 & 24.789 & 10.709 & & \\
\hline \multirow{2}{*}{ IL6 } & day 1 & 57.053 & 48.714 & \multirow{2}{*}{0.000} & \multirow{2}{*}{$Z=-4.033$} \\
\hline & day 4 & 36.026 & 12.980 & & \\
\hline \multirow{2}{*}{ IL17A } & day 1 & 8.342 & 10.850 & \multirow{2}{*}{0.925} & \multirow{2}{*}{$\mathrm{U}=-0.094$} \\
\hline & day 4 & 7.845 & 6.951 & & \\
\hline \multirow{2}{*}{ IL10 } & day 1 & 8.098 & 4.9412 & \multirow{2}{*}{0.000} & \multirow{2}{*}{$\mathrm{U}=-4.032$} \\
\hline & day 4 & 6.098 & 1.979 & & \\
\hline \multirow{2}{*}{$\mathrm{TNF} \alpha$} & day 1 & 12.872 & 3.502 & \multirow{2}{*}{0.006} & \multirow{2}{*}{$\mathrm{U}=-2.748$} \\
\hline & day 4 & 10.234 & 2.968 & & \\
\hline
\end{tabular}

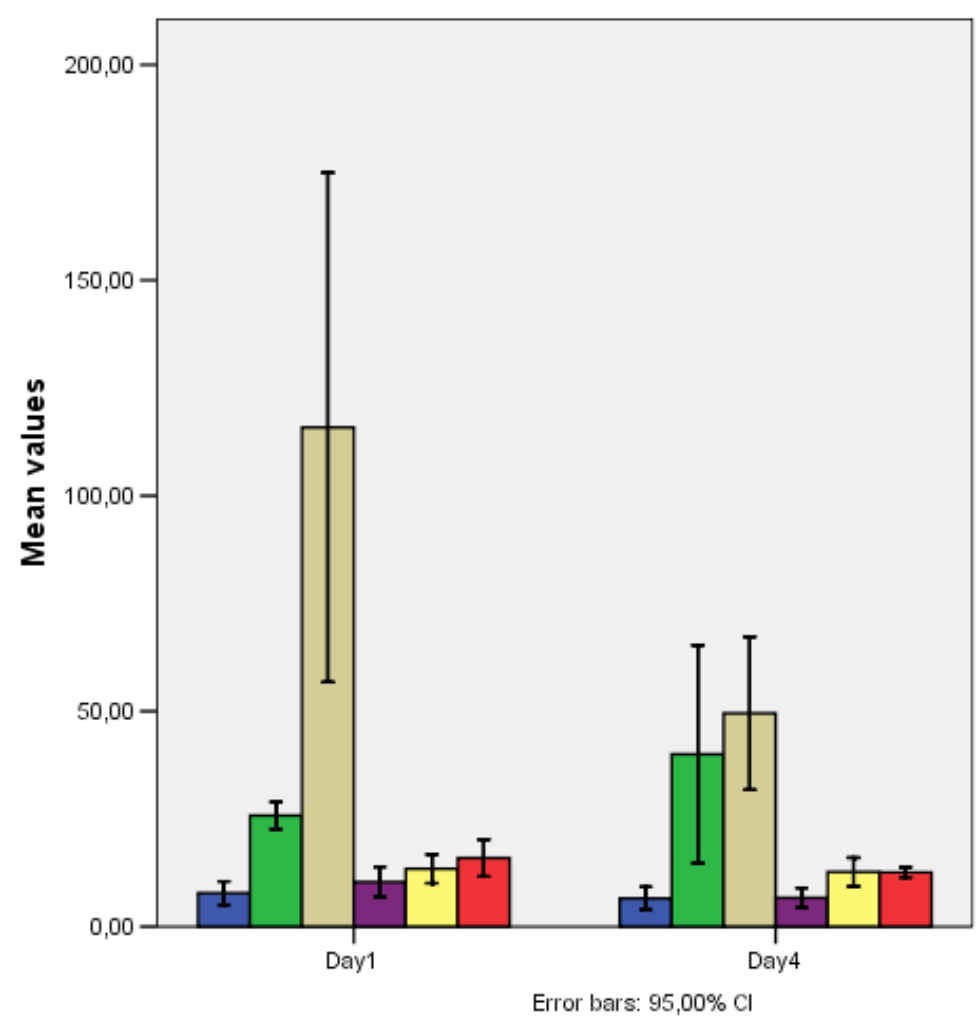

Fig.1. Serum concentration of interleukins on admission and on day 4 of hospitalization 
IL-4 ( $\mathrm{p}=0.056$, cut-off $=18.89 \mathrm{pg} / \mathrm{mL})$ and IL-10 $(p=0.07$, cut-off $=3.783 \mathrm{pg} / \mathrm{mL})$ tend to reach statistical significance. The ROC curve analysis for adverse outcome prediction on day 1 is shown in figure 2 .

The multivariate analysis of all combined interleukins on day 1 showed that there was a predictive model regarding adverse outcomes, AUC $=0.887$ with CI95\% [0.760, 0.913] $(\mathrm{p}=0.001)$. The model explains $87 \%$ of the variation of the outcomes.

We then studied if any of the interleukins were predictive for adverse outcomes on day 4. None reached statistical significance (figure 3 ).

Regarding the impact on adverse outcomes, the multivariate analysis of all combined interleukins has shown that the model explains roughly $48 \%$ of the variation in the outcomes (Wald chi2 test $(\mathrm{p}=0.025)$, Cox\&Snell $(\mathrm{R} 2=0.304)$, Nagelkerke $(\mathrm{R} 2=0.481)$ and Hosmer and Lameshow Test ( $\mathrm{p}=0.42$ ) were used). In multiple regression, using ENTER method, for adverse outcomes as the dependent variable, only IL- $1 \beta$ and IL- 6 had statistical significance on day 4 (table 3 ).

We questioned what happened between day 1 and day 4, so we studied the behaviour of each interleukin in every case. The behaviour of IL$1 \beta$ differed from day 1 to day 4 in every patient (Marginal Homogeneity Test $=286.64, \mathrm{p}=0.08$ ). Therefore, we applied Wilcoxon Signed Ranks test $(\mathrm{z}=-4.685, \mathrm{p}=0.001)$ which indicated that IL$1 \beta$ values decreased from day 1 to day 4 in most patients. The exceptions were 2 patients, from which 1 died and the other was aggravated. IL-6 had similar behaviour (Marginal Homogeneity Test $p=0.042$; Wilcoxon Signed Ranks Test $p=$

\section{ROC Curve}
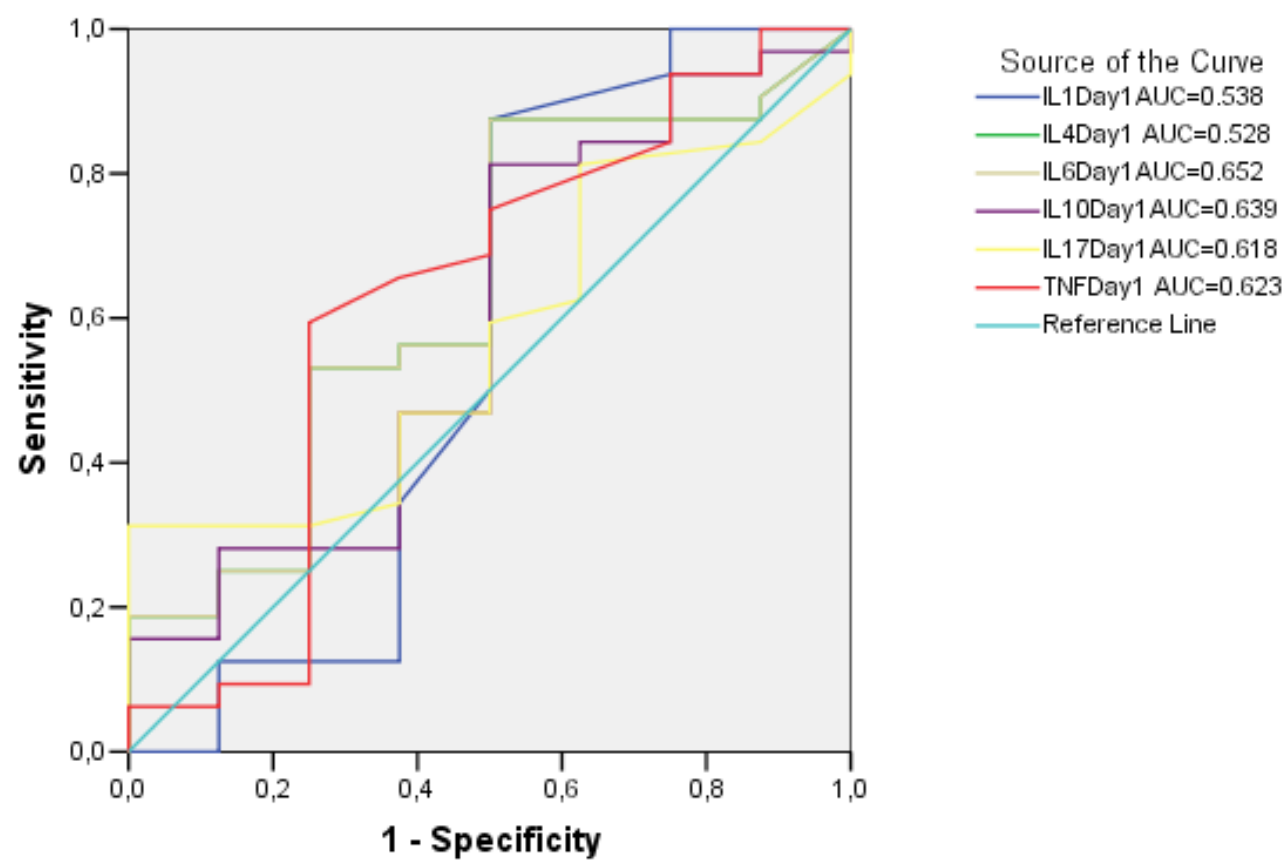

Fig.2. The ROC curve analysis for adverse outcome prediction on day 1 


\section{ROC Curve}

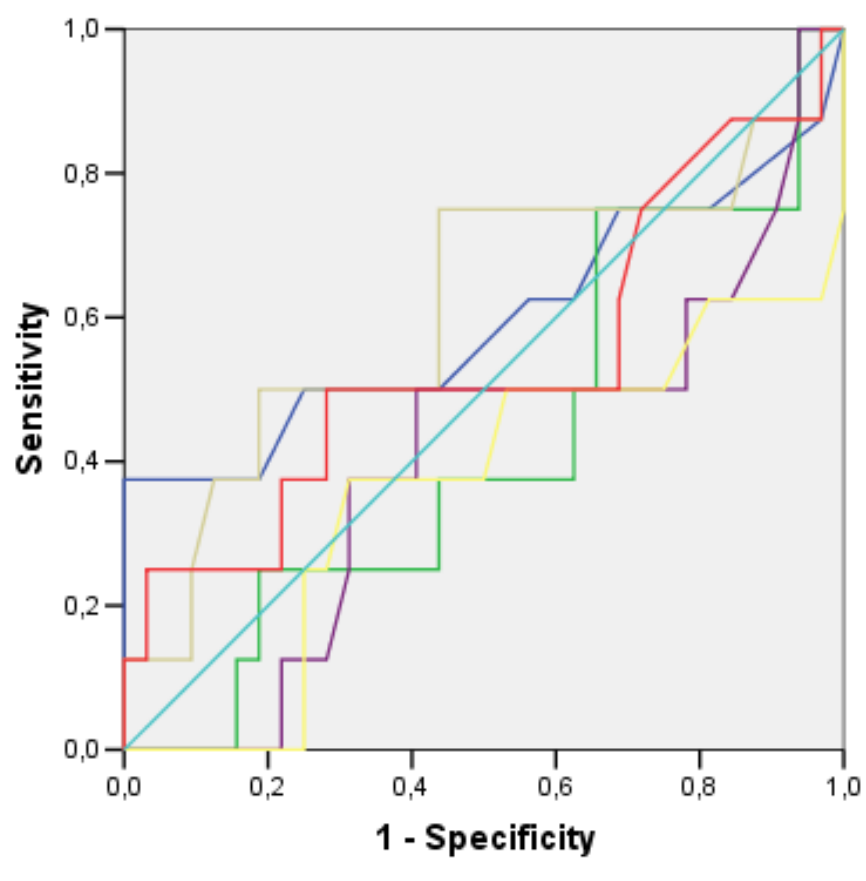

Source of the Curve

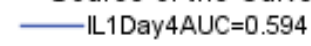

IL4Day4 AUC $=0.574$

IL6Day4 AUC $=0.613$

IL10Day4 AUC $=0.594$

IL17Day4 AUC $=0.653$

TNFDay4 AUC $=0.541$

Reference Line

Fig.3. The ROC curve analysis for adverse outcome prediction on day 4

0.003), except for 7 patients, from which 1 relapsed and only one had severe increase of the value on day 4 and he was the one who died. IL-10 had also a similar behaviour (Marginal Homogeneity Test $\mathrm{p}=0.049$, Wilcoxon Signed Ranks Test $\mathrm{p}=0.001$ ), with 6 exceptions, from which 1 died and 2 relapsed. IL-4 had opposite behaviour, meaning the values increased on day
4 (marginal Homogeneity Test $\mathrm{p}=0.03$, Wilcoxon Signed Ranks Test $\mathrm{p}=0.001$ ), but there were 13 exceptions, from which only 2 had relapses.

\section{Discussion}

Epidemiological studies tried to find the impact of age and gender on CAP caused by different

Table 3. The general multivariable logistic model for predicting mortality

\begin{tabular}{lccccc}
\hline Variable & Coefficient(B) & S. E. & P & Adjusted OR & 95\% CI for OR \\
\hline IL1_D4 & 2.409 & 1.529 & 0.015 & 11.122 & 0.556 to 22.49 \\
\hline IL4_D4 & -0.216 & 0.156 & 0.166 & 0.421 & 0.593 to 1.094 \\
\hline IL6_D4 & 0.016 & 0.013 & 0.027 & 0.978 & 0.991 to 1.043 \\
\hline IL10_D4 & -0.056 & 0.279 & 0.840 & 1.304 & 0.547 to 1.634 \\
\hline IL17_D4 & -0.040 & 0.148 & 0.787 & 1.362 & 0.719 to 1.284 \\
\hline TNF_D4 & -0.851 & 0.546 & 0.119 & 0.975 & 0.147 to 1.245 \\
\hline Constant & 0.701 & 4.994 & 0.888 & 2.016 & 0.801 to 3.786 \\
\hline
\end{tabular}


pathogens. They found that incidence rates were higher in males and increased by age [13]. Our findings are similar to that. Studies reported significant variations in mean LOS for patients with CAP in Europe and United States of America $[14,15,9]$. Rozenbaum M. et al reported a mean LOS of 6.7 days, while Garau J. et al found a mean LOS of 11.5 days. Our study population had a mean LOS of 8.5 days, which was shorter than in most similar cohorts in Europe. The etiology was found in only $25 \%$ of the cases, which was lower than in other reports. We used CURB-65, PSI, and SMART-COP scoring systems to predict mortality and the need for IRVS, at admission. The majority of our patients were included in PSI class risk 4 and 5 and CURB-65 group 2 and 3, but only 1 patient died. Regarding the need for IRVS calculated with SMART-COP, most of our cases had low risk.

This study aimed to analyze the relationship between pro and anti-inflammatory cytokines in the course of CAP, trying to bring a contribution to the knowledge of the disease, to a better understanding of its immunopathogenesis. Many studies have analyzed interleukin levels at a single time point, but dynamic changes in cytokines may have greater clinical value.

Similar to others, we have found no relationship between interleukin values and gender or age $[1,7]$. Bacci et al found an association between TNF- $\alpha$ and gender, and IL-1 was correlated with age, but the sample size was small, with only 26 subjects [16].

Previous studies found that systemic levels of IL-1 $\beta$, IL- 6 and TNF- $\alpha$ are usually elevated in patients with CAP, but they analyzed cytokines serum levels by only one determination [3,17]. G. Antunes et al showed a temporal pattern of these cytokines, with the serum levels declined rapidly over 5 days and postulated that pro-inflammatory cytokines were associated with poor prognosis in CAP [1]. M. Holub et al found that elevated levels of IL- 6 and TNF- $\alpha$ decreased within 3 days after admission, to levels similar to that of healthy subjects [18]. Similar with these findings, we found a decrease in the serum levels of IL-1 $\beta$, IL- 6 and TNF- $\alpha$ within 4 days of antibiotherapy, acting as acute phase proteins. One explanation is the start of antibiotic treatment, and, in some cases, corticotherapy, which reduces the inflammatory response. In our study, similar to others [16], we found correlations between pro-inflammatory cytokines IL-1 $\beta$, IL-6 and TNF- $\alpha$. The statistical model we used showed that high serum levels of IL- 6 on day 1 associated with adverse outcomes consisted in relapses or death after 90 days of discharge. Similarly, Bacci $\mathrm{M}$ et al demonstrated that serum levels of IL- 6 and TNF- $\alpha$ were correlated with worse outcomes [16]. There are several studies postulating that IL-6 is a marker of severity in CAP both in adults and children [7,19,20,21].

IL-4 has been best studied in the pathogenesis of asthma [22]. A recent study by M.J Giuffrida et al showed that there was no difference in serum levels of IL-4 and other cytokines studied, in asthmatic and non-asthmatic patients with bacterial, viral or mixed lung infection [23]. However, there are limited data regarding systemic levels of IL-4 in adults with CAP, most studies being concentrated on paediatric population and the role in the immune response against Mycoplasma pneumonia [6,24,25]. M.S Paats et al found that levels of IL-4 and IL-17A could not be detected in serum nor in bronchoalveolar lavage fluid (BAL) of the patients with CAP [7]. Unlike them, we found detectable serum levels of IL-4 on admission in almost all of our patients, with 2 exceptions, and in 27 cases their levels increased within 4 days of treatment. From the rest, 2 had relapses, and the others had only slightly decreased in their IL-4 values. Out of 38 patients, only 2 were known to have asthma, controlled at the time of hospital admission. Our results reinforce the idea that the immune response during bacterial infection is probably similar to the 
early phases of the immune response in asthma [23]. We also found a positive correlation between IL-4, IL-6, IL-17A and IL-10 that can be explained by a common origin, meaning T-helper cells. [26]

Regarding IL-10, we found an opposite behaviour to IL-4, paradoxically, the decline of values from day 1 to day 4, acting as an acute phase biomarker, consistent with previous reports $[1,3,5,7]$. Similar to these studies, we found a correlation between IL-6 and IL-10. A possible explanation for the different behaviour of IL-10 compared to IL-4 could be that they have different dynamics. Also, at the time of admission, patients were in different stages of pneumonia, a possible explanation for their differences.

It has been proven that IL-17A has a protective role in host defense against airway pathogens in mice, but it is also involved in the local and systemic immune response in human pneumonia $[27,28]$. In a recent study, F. Higa et al postulated that this cytokine might play a role in legionnaire disease [29]. M.S. Paats et al studied the systemic and local involvement of IL-17A in CAP, among other cytokines, but its levels in both BAL fluid and serum of patients or healthy controls were not found [7]. In our study, we found detectable levels of IL-17A in 38 patients, behaving mostly as pro-inflammatory cytokines, with decreasing levels from day 1 to day 4, although it did not reach statistical significance. Also we found a correlation between IL-17A, IL-6 and surprisingly IL-10, reinforcing the pro-inflammatory effect.

In this study we found a relationship between IL6 , IL-1 $\beta$, IL-4 and IL-10 and adverse outcomes (relapses and mortality). However, we have not found a correlation between cytokine levels and PSI risk class, partly because of the small number of subjects studied and also because we evaluated the severity scores only at admission. Endeman et al have also found no association between cytokine levels and this severity score
[5]. Kellum et al described the systemic cytokine response to infection and severe sepsis in a large cohort of patients with CAP, suggesting that individuals with high serum levels of both pro and anti-inflammatory cytokines had an increased risk of severe sepsis and death [30].

The present study has some limitations that should be considered. Firstly, the number of patients included was small, although we obtained relevant results for CAP. Secondly, we searched for systemic response only, not local immune response. Ideally, both responses should have been studied. However, many of the prognostic scores in CAP relate to the systemic rather than pulmonary effects of the biomarkers [31]. Another limitation is the prior prescription of antibiotics in $20 \%$ of our patients, which has certainly modulated the inflammatory response and cytokine expression. Also, the etiology was established in only 10 cases, which made it impossible to carry out statistical tests demonstrating the relationship between cytokines and etiology.

\section{Conclusion}

In conclusion, based on a comprehensive analysis of the systemic cytokine response in CAP, we have proved that IL- 6 is a predictive marker for adverse outcomes and should be routinely analyzed at admission. IL-17A and IL-10 behave like pro-inflammatory cytokines, underlining the fact that the dynamic of these cytokines depends on several factors, such as the time of admission and prior usage of antibiotics. Although the number of the patients was small, we demonstrated a model in which all the studied combined interleukins had an impact on adverse outcomes.

\section{Authors' contribution}

RET (Conceptualization; Investigation; Project administration; Writing - original draft) IBN (Data curation; Investigation; Validation) LB (Data curation; Formal analysis; Investiga- 
tion; Validation)

TLP (Conceptualization; Supervision; Writing review \& editing)

VC (Conceptualization; Project administration; Supervision; Writing - review \& editing)

LMS (Conceptualization; Data curation; Formal analysis; Validation)

MSL (Conceptualization; Methodology; Supervision; Visualization; Writing - review \& editing)

\section{Disclosure of interest}

The authors declare no conflict of interests.
Abbreviations
$\mathrm{CAP}=$ community-acquired pneumonia
$\mathrm{IL}=$ interleukin
$\mathrm{TNF}=$ tumor necrosis factor
IDSA = Infectious Diseases Society of America
LOS= length of stay
ACCP/SCCM= American College of Chest Phy-
sicians/Society of Critical Care Medicine
ELISA $=$ Enzyme linked immunosorbent assay
$\mathrm{ROC}=$ receiver operator curve
$\mathrm{BAL}=$ bronchoalveolar lavage fluid

\section{References}

1. Antunes G, Evans S, Lordan J, Frew A. Systemic cytokines levels in community-acquired pneumonia and their association with disease severity. Eur Respir J 2002;20:990-995. DOI: 10.1183/09031936.02.00295102

2. Community-Acquired Pneumonia (CAP): Practice Essentials, Overview, Etiology of Community-Acquired Pneumonia [Online]. [2017] [cited 29 April 2018]; Available from: URL: https://emedicine.medscape. com/article/234240-overview

3. Bordon J, Aliberti S, Fernandez-Botran R, Uriarte $\mathrm{S}$, Rane M, Duvvuri P, et al. Understanding the roles of cytokines and neutrophil activity and neutrophil apoptosis in the protective versus deleterious inflammatory response in pneumonia. Int J Infect Dis 2013;17(2):e76-e83. DOI: 10.1016/j.ijid.2012.06.006

4. Fernandez-Botran R, Wiemken T, Kelley R, Peyrani P, Bordon J, Cavallazzi R, et al. Analysis of the local and systemic cytokine response profiles in patients with community-acquired pneumonia. Relationship with disease severity and outcomes. Univ Louisville J Respir 2017;1(3):13-20. DOI: 10.18297/jri/vol1/iss3/5/

5. Endeman H, Meijvis S, Rijkers G, van Velzen-Blad H, van Moorsel C, Grutters J, et al. Systemic cytokine response in patients with community-acquired pneumonia. Eur Respir J 2011;37(6):1431-1438. DOI: 10.1183/09031936.00074410

6. Wang RS, Jin HX, Shang SQ, Liu XY, Chen SJ, Jin ZB. Associations of IL-2 and IL-4 Expression and Polymorphisms with the Risks of Mycoplasma pneumoniae Infection and Asthma in Children. Arch Bronconeumol 2015;51(11):571-8. DOI: 10.1016/j.arbres.2014.11.004

7. Paats M, Bergen I, Hanselaar W, Groeninx van Zoelen E, Hoogsteden H, Hendriks R, et al. Local and systemic cytokine profiles in nonsevere and severe community-acquired pneumonia. Eur Respir J 2013;41(6):137885. DOI: $10.1183 / 09031936.00060112$

8. Lorè N, Bragonzi A, Cigana C. The IL-17A/IL-17RA axis in pulmonary defence and immunopathology. Cytokine Growth Factor Rev 2016;30:19-27. DOI: 10.1016/j.cytogfr.2016.03.009

9. Mandell LA, Wunderink RG, Anzueto A, Bartlett JG, Dean NC, Whitney CG, et al. Infectious Diseases Society of America/ American Thoracic Society Consensus Guidelines on the Management of Community-Acquired Pneumonia in Adults. Clin Infect Dis 2007;44:S27-S72. DOI: 10.1086/511159

10. Eldaboosy SA, Halima KM, Shaarawy AT, Kanany HM, Elgamal EM, El-Gendi AA, et al. Comparison between CURB-65, PSI, and SIPF scores as predictors of ICU admission and mortality in community-acquired pneumonia. The egyptian journal of critical care medicine 2015;3(2):37-44. DOI: 10.1016/j.ejccm.2015.10.001

11. [Online]. 2018 [cited 26 July 2018]. Available from: URL: https://lifeinthefastlane.com/wp-content/uploads/2010/07/etg-smartcop.

12. Bone R, Balk R, Cerra F, Dellinger R, Fein A, Knaus W, et al. Definitions for Sepsis and Organ Failure and Guidelines for the Use of Innovative Therapies in Sepsis. Chest 1992;101(6):1644-1655. DOI: 10.1378/ chest.101.6.1644

13. Gutiérrez F, Masiá M, Mirete C, Soldán B, Carlos Rodríguez J, Padilla $\mathrm{S}$, et al. The influence of age and gender on the population-based incidence of community-acquired pneumonia caused by different microbial pathogens. J Infect 2006;53(3):166-174. DOI: 10.1016/j.jinf.2005.11.006

14. Rozenbaum M, Mangen M, Huijts S, van der Werf T, Postma M. Incidence, direct costs and duration of hospitalization of patients hospitalized with community acquired pneumonia: A nationwide retrospective claims database analysis. Vaccine 2015;33(28):3193-9. DOI: 10.1016/j.vaccine.2015.05.001 
15. Garau J, Baquero F, Pérez-Trallero E, Pérez J, Martín-Sánchez A, García-Rey C, et al. Factors impacting on length of stay and mortality of community-acquired pneumonia. Clin Microbiol Infect 2008;14(4):322-329. DOI: $10.1111 / \mathrm{j} .1469$ 0691.2007.01915.x

16. Bacci M, Leme R, Zing N, Murad N, Adami F, Hinnig $P$, et al. IL- 6 and TNF- $\alpha$ serum levels are associated with early death in community-acquired pneumonia patients. Braz J Med Biol Res 2015;48(5):427-432. DOI: 10.1590/1414-431X20144402

17. Puren A, Feldman C, Savage N, Becker P, Smith C. Patterns of Cytokine Expression in Community-Acquired Pneumonia. Chest 1995;107(5):1342-9. DOI: 10.1378/ chest.107.5.1342

18. Holub M, Lawrence D, Andersen N, Davidová A, Beran $\mathrm{O}$, Marešová $\mathrm{V}$, et al. Cytokines and Chemokines as Biomarkers of Community-Acquired Bacterial Infection. Mediators Inflamm 2013;2013:1-7. DOI: $10.1155 / 2013 / 190145$

19. Christ-Crain M, Opal S. Clinical review: The role of biomarkers in the diagnosis and management of community-acquired pneumonia. Crit Care 2010;14(1):203. DOI: $10.1186 / \mathrm{cc} 8155$

20. Vasconcellos Â, Clarêncio J, Andrade D, Cardoso MA, Barral A, Nascimento-Carvalho CM. Systemic cytokines and chemokines on admission of children hospitalized with community-acquired pneumonia. Cytokine 2018;107:1-8. DOI: 10.1016/j.cyto.2017.11.005

21. Khattab A, El-Mekkawy M, Shehata A, Whdan N. Clinical study of serum interleukin-6 in children with community-acquired pneumonia. Gaz Egypt Paediatr Assoc 2018;66(2):43-48. DOI: 10.1016/j.epag.2018.03.003

22. Chai R, Liu B, Qi F. The significance of the levels of IL-4, IL-31 and TLSP in patients with asthma and/ or rhinitis. Immunotherapy 2017;9(4):331-337. DOI: 10.2217/imt-2016-0131

23. Giuffrida M, Valero N, Mosquera J, Duran A, Arocha F, Chacín B, et al. Increased Systemic Cytokine/Chemokine Expression in Asthmatic and Non-asthmatic Pa- tients with Bacterial, Viral or Mixed Lung Infection. Scand J Immunol 2017;85(4):280-290. DOI: 10.1111/ sji. 12532

24. Medjo B, Atanaskovic-Markovic M, Nikolic D, Radic $\mathrm{S}$, Lazarevic I, Cirkovic I, et al. Increased Serum Interleukin-10 but not Interleukin-4 Level in Children with Mycoplasma pneumonia pneumonia. J Trop Pediatr 2016;63(4):1-7.

25. Xu X, Li X, Liu J, Wu L, Chen Z. Serum cytokine profile contributes to discriminating $M$. pneumoniae pneumonia in children. Cytokine 2016;86:73-78. DOI: 10.1016/j.cyto.2016.07.018

26. Chaudhry H, Zhou J, Zhong Y, Ali MM, McGuire F, Nagarkatti P, et al. Role of cytokines as a double-edged sword in sepsis. In Vivo 2015;27(6):669-84.

27. Kurata S, Osaki T, Yonezawa H, Arae K, Taguchi H, Kamiya S. Role IL-17A and IL-10 in the antigen induced inflammation model by Mycoplasma pneumoniae. BMC Microbiol 2014;14(1): 156. DOI: 10.1186/14712180-14-156

28. Paats M, Bergen I, Hanselaar W, van Zoelen E, Verbrugh $\mathrm{H}$, Hoogsteden $\mathrm{H}$, et al. T helper 17 cells are involved in the local and systemic inflammatory response in community-acquired pneumonia. Thorax 2013;68(5):468474. DOI: 10.1136/thoraxjnl-2012-202168

29. Higa F, Haroon A, Iha Y, Tasato D, Nakamura H, Kinjo $\mathrm{T}$, et al. Interleukin-17A in Legionella pneumonia: A Retrospective Study. Jpn J Infect Dis 2015;68(2):148150. DOI: 10.7883 /yoken.JJID.2014.171

30. Kellum JA, Kong L, Fink MP, Weissfeld LA, Yealy $\mathrm{MD}$, Fine J, et al. Understanding the Inflammatory Cytokine Response in Pneumonia and Sepsis: results of the Genetic and Inflammatory Markers of Sepsis (GenIMS) Study. Arch Intern Med 2007;167(15):1655-63. DOI: $10.1001 /$ archinte.167.15.1655

31. Marti C, Garin N, Grosgurin O, Poncet A, Combescure $\mathrm{C}$, Carballo S, et al. Prediction of severe community-acquired pneumonia : a systematic review and meta-analysis. Crit Care 2012;16(4):R141. DOI: 10.1186/ cc11447 